\title{
Entrenamiento conductual del uso correcto del condón femenino en adolescentes: un estudio aleatorizado
}

\author{
Behavioral training of the correct use of female condom \\ in adolescents: $A$ randomized study
}

\author{
Edgar Landa-Ramírez, Sandra Itzel Quiñones-Sandoval, \\ Alma Delia Flores-Ordaz, Aime Edith Martínez-Basurto \\ y Juan José Sánchez-Sosa ${ }^{1}$
}

\begin{abstract}
RESUMEN
El objetivo del estudio fue evaluar el efecto de dos tipos de entrenamiento conductual en el desarrollo de habilidades instrumentales y verbales para el uso correcto del condón femenino en adolescentes de la Ciudad de México. Un segundo objetivo buscó identificar los principales errores cometidos por los participantes al colocar el condón femenino. La metodología incluyó una comparación de dos grupos, en los que participaron 116 adolescentes asignados de manera aleatoria a uno de ellos. Se registraron por observación directa las habilidades instrumentales y verbales para colocar el condón en modelos de vaginas de entrenamiento en evaluaciones pre, post y seguimiento a tres meses. Ambos entrenamientos incrementaron la frecuencia de respuestas correctas en la postevaluación y en el seguimiento, incremento que fue estadísticamente significativo. Se describen algunos errores en la colocación del condón. Ambos tipos de entrenamiento fueron efectivos para desarrollar las habilidades requeridas para el uso correcto del condón femenino. Se discuten las limitaciones de los datos y las implicaciones para investigaciones futuras.
\end{abstract}

Palabras clave: Entrenamiento conductual; Adolescentes; Condón femenino; Errores al usar el condón.

\begin{abstract}
The objective of the present study was to examine the effects of two training techniques: a) behavioral skills training and b) modeling, on instrumental and verbal behaviors while properly placing female condoms. A secondary objective involved identifying specific types of errors while displaying the condom-placing behavioral sequence. The study's methodology involved 116 adolescents randomly assigned to a two-group design. Direct behavioral observation procedures were implemented to record both instrumental and verbal responses while participants placed female condoms on model training vaginas. Measurements included pretest, posttest and a three-month follow up. Results revealed both procedures produced statistically significant increases from pretest to both posttest and follow up. Some errors while placing the condom included not using the proper finger combination to hold the condom and difficulties finding a comfortable position to ease its placement. Thus, we conclude that both behavioral training procedures were effective to teach proper placing of female condoms in teenagers. The study's findings are discussed in the context of their applied preventive contribution as well as limitations and suggestions for future studies.
\end{abstract}

Key words: Behavioral training; Adolescents; Female condom; Errors in condom use.

\footnotetext{
${ }^{1}$ Programa de Psicología de la Salud y Medicina Conductual, Facultad de Psicología, Universidad Nacional Autónoma de México, Av. Universidad 3004, Del. Coyoacán, 04510 Ciudad de México, México, tel. (55)56-22-23-32, correo electrónico: edgar_landa_ramirez@yahoo.com.mx. Artículo recibido el 27 de enero y aceptado el 22 de marzo de 2017.
} 


\section{INTRODUCCIÓN}

$\mathrm{L}$ opez, Stockton, Chen, Steiner y Gallo (2014) han documentado la eficacia de los métodos de barrera -en particular el condón femenino- para evitar embarazos no deseados y prevenir infecciones de transmisión sexual (ITS), como gonorrea, clamidia y sífilis (Vijayakumar, Mabude, Smit, Beksinska y Lurie, 2006). Su uso también ayuda a prevenir la infección por el virus de inmunodeficiencia humana (VIH) (Campbell et al., 2011) y se le considera medianamente efectivo para prevenir el contagio del virus de papiloma humano (Shepherd, Frampton y Harris, 2011). La Administración de Alimentos y Medicamentos de Estados Unidos (Food and Drug Administration) aprobó en marzo de 2009 la segunda generación del condón femenino (CF2) en virtud de que su efectividad no mostraba ser inferior a la de la primera generación del dispositivo; hoy, se le sigue considerando como una protección efectiva contra los citados problemas de salud (Beksinska et al., 2013).

$\mathrm{Al}$ igual que ocurre con el condón masculino, la efectividad del CF2 para prevenir los problemas mencionados depende en gran medida de su uso correcto y consistente (Sanders et al., 2012), por lo que adquiere especial relevancia desarrollar líneas de investigación para fomentar dicho uso (Mantell et al., 2011). En el caso del CF2, los resultados de diversos estudios experimentales sugieren que los entrenamientos conductuales son efectivos para impulsar su uso correcto en diversas poblaciones adultas (Campbell et al., 2011; Choi et al., 2008; Hoffman, Exner, Leu, Ehrhardt y Stein, 2003; Kalichman, Williams y Nachimson, 1999; Mantell et al., 2014; Van Devanter et al., 2002; Witte et al., 2006; Zimmers, Privette, Lowe y Chappa, 1999). Sin embargo, algunas características de esos estudios podrían constituir limitaciones para generalizar sus resultados a la población adolescente; por ejemplo, la mayoría de los mismos se han hecho solo con mujeres, mayores de edad, con vida sexual activa, en riesgo de adquirir ITS y que asistían a centros de planificación familiar o a servicios de salud de alguna institución de educación superior (Campbell et al., 2011; Choi et al., 2008; Schuyler et al., 2016).
Lo anterior representa un problema importante por sus implicaciones demográficas de riesgo. Por ejemplo, en Estados Unidos los adolescentes inician su vida sexual a los 14 años en promedio y alrededor de la mitad habrá tenido tres parejas sexuales antes de cumplir los 19 años (Cavazos-Rehg et al., 2009; Liu et al., 2015). En la zona central de México se han documentado resultados similares en cuanto al inicio de la vida sexual. Una encuesta hecha a 13,293 adolescentes reveló que los hombres comienzan su vida sexual a los 13 años, y las mujeres a los 14 , y únicamente $35 \%$ de los entrevistados reportaron haber utilizado condón (masculino) en su primer encuentro sexual (Tapia et al., 2004).

Entrenar en el uso correcto del CF2 podría ser una opción alterna o complementaria para proteger a los adolecentes de embarazos no planeados y contagios al comienzo de su vida sexual. Sin embargo, la falta de datos sobre los efectos de entrenamientos conductuales particulares no permite hacer recomendaciones sobre el tipo de intervención que conviene usar y adaptar a las necesidades de los jóvenes.

A pesar de las limitaciones descritas, hay estudios que muestran los efectos positivos de algunos programas de entrenamiento (Orr, Langefeld, Katz y Caine, 1996; Shepherd et al., 2010). Se han empleado dos estrategias conductuales para establecer esas habilidades en los adolescentes. La primera se enfoca a brindar información por medio de talleres o pláticas sobre la importancia del uso correcto del condón masculino, discutir la importancia de prevenir ITS, proporcionar información impresa en folletos y usar el modelamiento para mostrar su colocación. En estos entrenamientos, los adolescentes no manipulan el condón, sino que simplemente observan (Orr et al., 1996). En la segunda estrategia, además de brindar la información acerca del condón y la prevención de las ITS, se entrena a los adolescentes, mediante instrucciones, en los pasos a seguir para usar correctamente el dispositivo y manipular directamente los preservativos en penes de entrenamiento, retroalimentándolos por su desempeño eficaz (Shepherd et al., 2010). En el caso del condón masculino, el último tipo de entrenamiento ha tenido mejores efectos en el desarrollo de las habilidades necesarias para su uso correcto. 
Los datos anteriores permitirían suponer que los adolescentes también se pueden beneficiar de recibir un entrenamiento en la modalidad conductual a fin de que desarrollen habilidades para usar correctamente el CF2; sin embargo, la falta de evidencia específica no ha permitido valorar sus posibles efectos en esta población, lo que implica un vacío en la información nacional e internacional.

Debido a los beneficios potenciales de entrenar a esta población y a la carencia de estudios que incorporen adolescentes de edades cercanas al debut sexual (alrededor de los 14 años), se decidió llevar a cabo el presente estudio, cuyo objetivo fue evaluar el efecto de dos intervenciones conductuales (intervención por modelamiento e intervención de habilidades conductuales) en el desarrollo de las habilidades requeridas para el uso correcto del condón femenino en la población adolescente de la Ciudad de México. De igual forma, debido a que pocas investigaciones describen los errores que cometen las personas al colocar el condón (Baćak y Stulhofer, 2012; Sanders et al., 2012), se consideró indispensable describir los principales yerros de los participantes antes de recibir el entrenamiento y posteriormente al mismo.

\section{MÉTODO}

\section{Participantes}

Se realizó una junta informativa con los maestros y padres de familia de los alumnos del último año de secundaria, para comentar el propósito y alcances de la investigación y señalar la importancia de la firma del consentimiento informado para participar. El texto del consentimiento informado también reseñó los objetivos generales del estudio. Se informó a los participantes que, si lo deseaban, podían abandonar la investigación sin que hubiera ningún tipo de consecuencia. Todas las sesiones se llevaron a cabo en las aulas de la escuela.

Se invitó a participar a 189 estudiantes de ambos sexos, alumnos del tercer año de una secundaria pública de la Ciudad de México. De ellos, 69 no entregaron el formato de consentimiento informado firmado por sus padres o por ellos mismos, por lo cual no participaron en el estudio; cuatro estudiantes se rehusaron a participar, con lo que quedaron 116 estudiantes. Los participantes se seleccionaron de manera no probabilística accidental. En cuanto a las principales características sociodemográficas, participaron 58 mujeres y 58 hombres, mexicanos, solteros, de clase media baja y con una edad promedio de 14.1 años $($ D.E. $=.40)$. De ellos, 95\% eran de religión católica. Catorce refirieron haber comenzado ya su vida sexual $($ edad promedio $=13.4$ años; D.E. $=.72)$, doce de los cuales indicaron haberse protegido con condón masculino en su primer encuentro sexual y dos manifestaron no haberlo hecho. No hubo diferencias estadísticamente significativas en las condiciones sociodemográficas previas al entrenamiento a las cuales se pudiera atribuir los cambios observados en la variable dependiente en las postevaluaciones (Tabla 1).

Tabla 1. Características sociodemográficas de los participantes.

\begin{tabular}{|c|c|c|c|}
\hline \multirow{2}{*}{ Participantes } & $\begin{array}{l}\text { Entrenamiento } \\
\text { modelamiento }\end{array}$ & $\begin{array}{c}\text { Entrenamiento } \\
\text { habilidades conductuales }\end{array}$ & \multirow[t]{2}{*}{$p$} \\
\hline & 57 & 59 & \\
\hline Edad, media, desviación estándar (D.E.) & 14.16 (D.E.: .36) & 14.19 (D.E.: .43) & $.67^{\mathrm{A}}$ \\
\hline $\begin{array}{l}\text { Sexo } \\
\text { Mujeres } \\
\text { Hombres }\end{array}$ & $\begin{array}{l}26 \\
31 \\
\end{array}$ & $\begin{array}{l}32 \\
27\end{array}$ & $.35^{\mathrm{B}}$ \\
\hline $\begin{array}{l}\text { Debut sexual } \\
\text { Sí } \\
\text { Edad de debut } \\
\text { No } \\
\text { No contestó }\end{array}$ & $\begin{array}{c}5 \\
13.6 \text { años (D.E.: .5) } \\
42 \\
10\end{array}$ & $\begin{array}{c}9 \\
13.3 \text { (D.E..: .8) } \\
37 \\
13\end{array}$ & $.24^{\mathrm{A}}$ \\
\hline $\begin{array}{l}\text { Habilidades durante la preevaluación } \\
\text { Habilidades verbales } \\
\text { Habilidades instrumentales }\end{array}$ & $\begin{array}{c}2 \\
3.2 \text { (D.E.: } 1.7)\end{array}$ & $\begin{array}{l}2.3 \text { (D.E.: } 1.6) \\
3.1 \text { (D.E.: } 1.5 \text { ) }\end{array}$ & $\begin{array}{l}.28^{\mathrm{A}} \\
.82^{\mathrm{A}}\end{array}$ \\
\hline
\end{tabular}

D.E. $=$ Desviación estándar; $\mathrm{A}=\mathrm{U}$ de Mann-Whitney; $\mathrm{B}=$ Chi cuadrada. 


\section{Mediciones}

Habilidades para el uso del condón femenino: En concordancia con estudios previos, el uso correcto del condón femenino se determinó por medio de la observación directa hecha por psicólogos entrenados, quienes registraban las respuestas instrumentales y verbales de los participantes al colocar el condón femenino en un modelo de vagina de entrenamiento (Campbell et al., 2011). Se decidió diferenciar las habilidades en términos de respuestas instrumentales y verbales, pues ser ca- paz de referir cómo se debe poner el condón no significa que se haya desarrollado la habilidad de colocarlo correctamente, y viceversa. $\mathrm{Al}$ igual que en estudios previos, se retomaron los once pasos derivados de las recomendaciones para usar correctamente el CF2 sugeridas por la Female Health Company (2012). Se probó la factibilidad de aplicar los pasos en cinco participantes voluntarios, a partir de los cuales se vio la necesidad de separar las instrucciones del paso 11, convirtiéndolas en dos pasos, quedando así los doce pasos utilizados en el estudio (Tabla 2).

Tabla 2. Pasos para usar correctamente el condón femenino.

Pasos entrenados durante el estudio adaptados de la Female Health Company

1. Verifica la fecha de caducidad que aparece en la parte frontal o lateral del paquete.

2. Esparce el lubricante en el interior frotándolo con los dedos.

3. Para abrir el condón, rasga desde la flecha situada en el borde (flecha morada) y extráelo. No uses tijeras, cuchillos o los dientes para abrir el paquete.

4. Toma el anillo interno entre los dedos pulgar e índice.

5. Aprieta el anillo interior por los lados hasta que se junten y formen una punta.

6. Puedes insertar el condón de diferentes maneras. Encuentra una posición que te sea cómoda. Puedes estar sentada(o), en cuclillas o acostada(o).

7. Identifica los labios externos de la vagina y sepáralos.

8. Entonces, usa la punta en forma de " 8 " del anillo interno y empújalo dentro de la vagina. Usa la punta formada por la unión de los lados del anillo del condón ("8") para empujarlo dentro de la vagina.

9. Desliza el dedo índice o medio dentro del condón y empuja el anillo interior que está dentro de la vagina hasta donde sea posible. Asegúrate de que el condón se ajuste suavemente.

10. Sujeta bien el anillo exterior y mantenlo en un sitio mientras el pene se orienta dentro del condón. Cuando el pene esté dentro de él, ya no es necesario seguir sosteniendo el anillo exterior.

11. Para retirar el condón, toma el anillo exterior y tuércelo para mantener el semen adentro. Es mejor hacerlo antes de levantarse.

12. Quita suavemente el condón, envuélvelo en un pañuelo de papel y echa a la basura el paquete vacío.

En total, participaron tres psicólogos observadores, quienes recibieron un entrenamiento de un mes para llevar a cabo el registro observacional de las respuestas verbales e instrumentales a evaluar. A las respuestas correctas se les dio el valor de $1, \mathrm{y}$ de 0 a las incorrectas; al final de las evaluaciones, se sumaron los puntajes para obtener el total de respuestas correctas.

\section{Diseño y procedimiento}

Se trató de un estudio aleatorizado de dos grupos: Grupo 1, de habilidades conductuales $(\mathrm{n}=59)$ y Grupo 2, de modelamiento $(\mathrm{n}=57)$. Los participantes se asignaron de manera aleatoria simple en una proporción de 1:1. Se utilizó un programa computarizado para realizar la aleatorización (véase www.randomization.com). Se realizaron evaluaciones pre, post y seguimiento a los tres meses (Figura 1).

\section{Preevaluación}

Antes de iniciar los entrenamientos, se dio a cada participante individual un condón femenino y el modelo de vagina de entrenamiento en un cubículo escolar de aproximadamente 3 por 3 metros, con mobiliario habitual y en presencia de un psicólogo observador. Se les instruyó a mostrar y describir, de acuerdo con sus conocimientos, cómo se usaba correctamente el CF2. El observador registraba las conductas instrumentales y verbales mostradas 
Figura 1. Diagrama de flujo de los participantes durante el estudio.

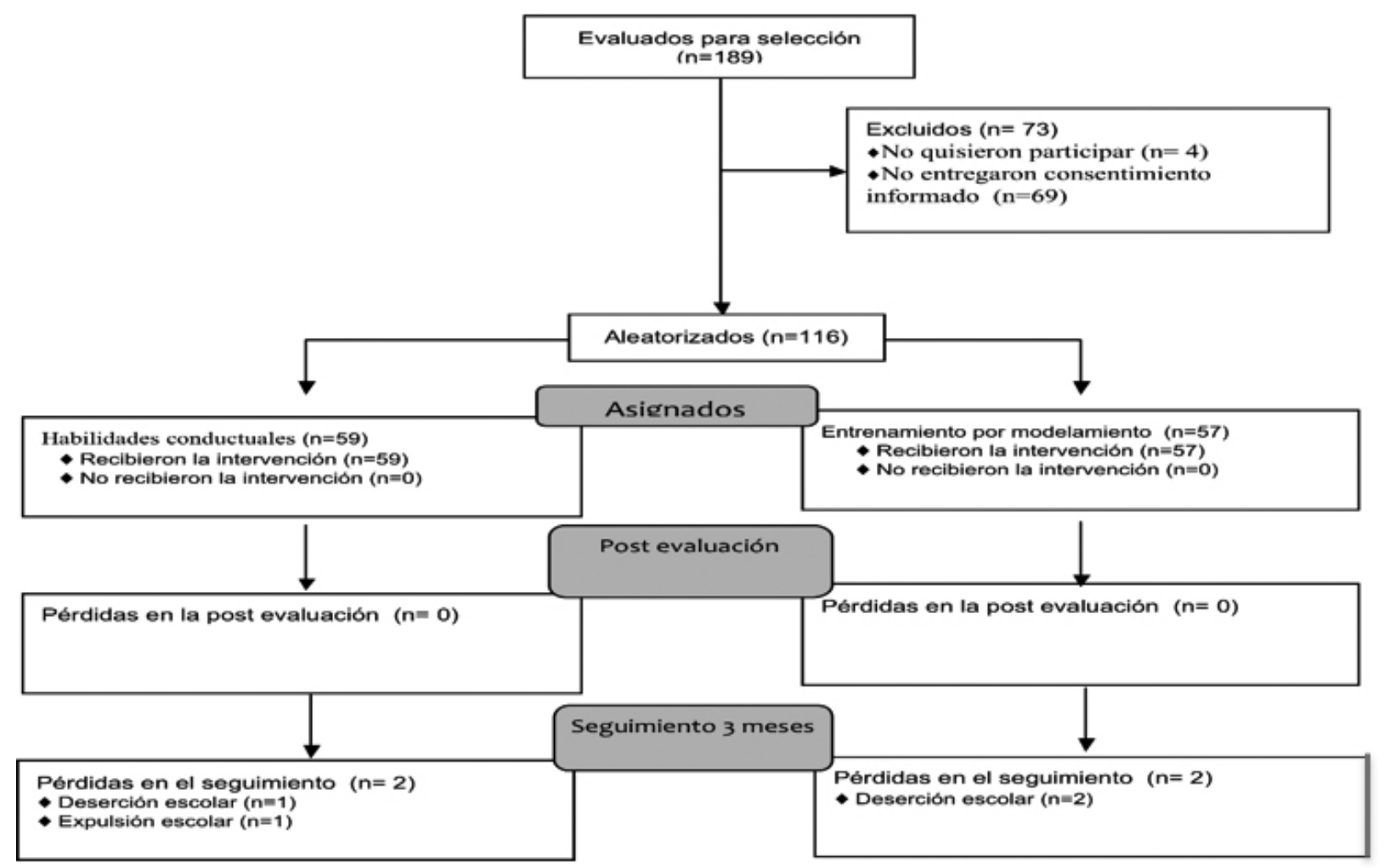

por los participantes, a quienes se les señaló que durante esta evaluación no se les daría retroalimentación por su desempeño.

\section{Entrenamiento}

Los entrenamientos se derivaron de una adaptación de las estrategias utilizadas en estudios previos, como los de Orr et al. (1996), Van Devanter et al. (2002) y Campbell et al. (2011). Se llevaron a cabo cuatro sesiones semanales de una hora cada una. La primera tuvo como propósito que los participantes identificaran los diversos cambios que ocurren durante la adolescencia y su impacto en el deseo sexual; la segunda, las principales enfermedades de transmisión sexual y los medios de contagio más importantes; la tercera, el impacto de los métodos de barrera en la protección con énfasis marcado en el impacto del CF2, y la cuarta, los doce pasos para usar el CF2 de manera correcta.

Las primeras tres sesiones se impartieron grupalmente en las aulas escolares. La cuarta sesión se hizo de manera individual a todos los que pertenecían al grupo de entrenamiento de habilidades conductuales. Para la enseñanza de los doce pasos, se proporcionó a cada participante un con- dón femenino y un modelo de vagina de entrenamiento, tras de lo cual se les dio retroalimentación descriptiva en cada uno de los pasos, hasta que los realizaron de manera correcta. El proceso duró 15 minutos en promedio.

En el caso de los participantes del grupo de modelamiento, uno de los investigadores, utilizando una vagina de entrenamiento y un CF2, mostraba cómo se debía de colocar correctamente el condón según los doce pasos. Se decidió integrar el modelamiento debido a que los programas únicamente informativos no han reportado efectos tan favorables en el desarrollo de las habilidades para usar correctamente el condón (Orr et al., 1996); dicho entrenamiento se impartía grupalmente y los adolescentes no manipulaban el CF2.

\section{Postevaluación y seguimiento}

Una semana posterior a haber completado el entrenamiento se hizo una evaluación de manera muy similar a la de la preevaluación; de igual forma, se realizó el seguimiento tres meses después. En estas mediciones, al igual que en la preevaluación, no se les dio retroalimentación a los participantes. Un psicólogo observador registró los 
comportamientos instrumentales y verbales de los participantes.

\section{Análisis de datos}

A fin de identificar homogeneidad en las varianzas y distribución normal, se aplicaron las pruebas de Levene y de Kolmogorov-Smirnov. Al no encontrarse una distribución normal, y dado el carácter nominal (correcto-incorrecto en frecuencias), se decidió utilizar la prueba U de Mann Whitney para contrastar las medianas de dos grupos independientes con libre distribución. De igual forma, se empleó el test de Wilcoxon para la contrastación de la mediana de dos muestras relacionadas con libre distribución. Finalmente, se aplicó la prueba chi cuadrada para contrastar los datos categóricos. Se consideró estadísticamente significativo el dato si la probabilidad asociada a las diferencias era $p \leq .05$.

\section{RESULTADOS}

Durante la preevaluación, los dos grupos mostraron escasas habilidades instrumentales y verbales en el uso correcto del condón femenino. El grupo que recibiría el entrenamiento conductual tuvo en promedio 2.3 pasos correctos de los 12 pasos posibles (2.3/12) (D.E.:1.6; mediana: 2) al describir la colocación del condón femenino, y 3.1/12 al mostrarlo instrumentalmente (D.E.:1.5; mediana: 3). El grupo que se expondría al modelamiento tuvo en promedio $2.7 / 12$ respuestas correctas al decir cómo se pone el condón femenino (D.E.:1.8; mediana: 3), y 3.2/12 al mostrarlo (D.E.:1.7; mediana: $3)$. No hubo diferencias estadísticamente significativas en las habilidades de los participantes en cada uno de los grupos durante la preevaluación (habilidades instrumentales: $p=<0.81$; habilidades lingüísticas: $p=<0.28$ ). Se encontró una diferencia estadísticamente significativas en la preevaluación debida al sexo, ya que las mujeres dieron un mayor número de respuestas verbales que los hombres en el grupo de modelamiento (mujeres: 3.4 D.E.:1.7; hombres: 1.8, D.E.: $1.4, p=<0.05$ ), dato que se retoma en la discusión.

Durante la postevaluación, ambos grupos incrementaron sus habilidades instrumentales y verbales sobre el uso correcto del condón femenino (Figura 2).

Figura 2. Promedio de respuestas correctas durante el estudio.
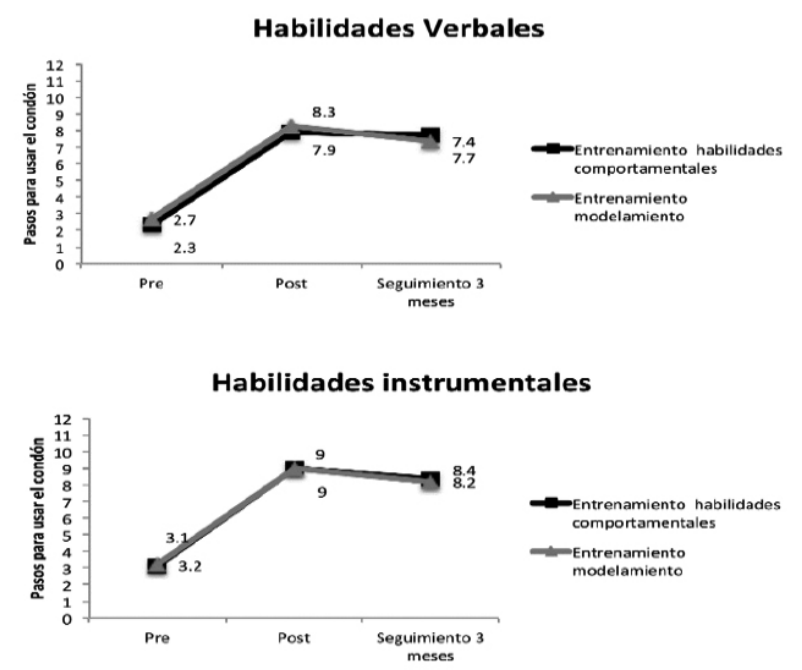

El grupo de entrenamiento conductual tuvo en promedio 7.9/12 respuestas correctas al decir cómo se coloca el condón femenino (D.E. = 1.8; mediana: 8 ) y $9 / 12$ al mostrarlo (D.E. $=1.3$; mediana: 9 ). El grupo de modelamiento tuvo en promedio $8.3 / 12$ respuestas correctas al decir cómo se pone el condón femenino (D.E. = 1.8; mediana: 9) y 9/12 al mostrar- lo (D.E. = 1.5; mediana: 9). Durante la postevaluación no hubo diferencias estadísticamente significativas entre ambos grupos $(\mathrm{Z}=-1.191, p=<.234)$. Al comparar las diferencias entre los valores totales de la preevaluación y la postevaluación de ambos grupos en habilidades instrumentales y verbales, se encontró que los dos mostraron diferencias estadís- 
ticamente significativas entre habilidades instrumentales $(\mathrm{Z}=-9.380, p=<.001 \mathrm{y}$ habilidades lingüísticas $(\mathrm{Z}=-9.286, p=<.001)$. En la postevaluación ya no hubo diferencias estadísticamente significativas en las habilidades verbales debidas al sexo.

De igual forma, durante el seguimiento a tres meses ambos grupos mantuvieron niveles similares en sus habilidades instrumentales y verbales sobre el uso correcto del condón femenino. El grupo de habilidades conductuales tuvo en promedio 7.7/12 respuestas correctas al decir cómo se coloca el condón femenino (D.E. $=2.6$; mediana: 7), y $8.4 / 12$ al mostrarlo (D.E. $=1.3$; mediana: 9). El grupo de modelamiento tuvo en promedio $7.4 / 12$ respuestas correctas al decir cómo se pone el condón femenino (D.E. = 1.8; mediana: 8) y 8.2/12 al mostrarlo (D.E. $=1.7$; mediana: 8 ). Una vez más, durante el seguimiento no se hallaron diferencias estadísticamente significativas entre ambos grupos ( $\mathrm{Z}=-1.191, p=<.234)$. Al comparar las diferencias entre los valores totales de la preevaluación y el seguimiento de ambos grupos en las habilidades instrumentales y verbales, se encontraron tuvieron diferencias estadísticamente significativas entre habilidades instrumentales $(\mathrm{Z}=-9.380, p=<.001)$ y habilidades verbales $(Z=-9.286, p=<.001)$. Igualmente, al comparar las diferencias entre los valores totales de la postevaluación y los del seguimiento de ambos grupos en las habilidades instrumentales y verbales, se encontró que los dos tuvieron diferencias estadísticamente significativas entre habilidades instrumentales $(Z=-3,510$ $p=<.001)$ y habilidades verbales $(\mathrm{Z}=-4.635$, $p=<.001)$. No hubo diferencias estadísticamente significativas debidas al sexo en el seguimiento.

\section{Errores en el uso del condón}

Durante la preevaluación, los participantes mostraron un alto porcentaje de errores para colocar el condón femenino (75.48\%; D.E.: 22.67). En las habilidades verbales, se equivocaron en promedio 78.2\% (D.E.: 19.90). Los únicos pasos que tuvieron un porcentaje de error menor o cercano a $50 \%$ fueron el paso $3(50.4 \%)$ y el $5(47.9 \%)$. En el caso de las habilidades instrumentales, el promedio de errores de los participantes fue de $72.7 \%$ (D.E.:23.95). En tres pasos hubo un porcentaje de error menor o cercano a $50 \%$ : el $3(23.1 \%)$, el 5 $(45.3 \%)$ y el $8(50.4 \%)$.

Durante la postevaluación, el porcentaje general de errores disminuyó a $28.4 \%$ (D.E.: 24.83), el de errores de habilidades verbales disminuyó a $31.69 \%$ (D.E.: 25.74) y el de errores de habilidades instrumentales disminuyó a $25.17 \%$ (D.E.: 23.43). A pesar de la disminución de los errores en las habilidades verbales, hubo tres pasos cuyos porcentajes de error se mantuvieron muy altos: el 4 (63.2\%), el 6 (73.5\%) y el 7 (63.2\%). Algo similar ocurrió en los errores referidos a las habilidades instrumentales, ya que tres pasos se mantuvieron altos: el $6(73.2 \%)$, el $7(56.4 \%)$ y el $10(50.4 \%)$.

Durante el seguimiento a tres meses, el porcentaje general de errores mostró un ligero aumento a $32.61 \%$ (D.E.: 27.07). El de errores de habilidades verbales aumentó a $36.31 \%$ (D.E.: 26.91) y el de errores de habilidades instrumentales mostró un pequeño incremento a $28.90 \%$ (D.E.: 26.73). Es de notarse que hubo cinco pasos en habilidades verbales cuyos porcentajes de error se mantuvieron altos: el $2(53.8 \%)$, el $4(58.1 \%)$, el $6(75.2 \%)$, el $7(82.1 \%)$ y el $10(51.3 \%)$. En cuanto a los errores de las habilidades instrumentales, tres pasos se mantuvieron altos en esta etapa: el 2 (49.6\%), el 6 (84.6\%) y el 7 (76.1\%) (Figura 3).

\section{DISCUSIÓN Y CONCLUSIONES}

Los resultados anteriores indican que ambas intervenciones aumentaron las habilidades verbales e instrumentales para el uso correcto del condón femenino. En el contexto de los estudios antecedentes, si bien muchos de ellos concluyen que las intervenciones conductuales aumentan las habilidades para usar el condón femenino, no ocurre así con aquellas en que los participantes no se exponen a la manipulación del condón (Campbell et al., 2011; Choi et al., 2008; Hoffman et al., 2003; Kalichman et al., 1999; Van Devanter et al., 2002; Witte et al., 2006; Zimmers et al., 1999). Es probable que el efecto positivo logrado en el presente estudio se derive de que en la intervención se utilizaron técnicas de modelamiento mediante las cuales se proporcionaba información específica y concreta acerca de los pasos para colocar correctamente el CF2 y se demostraban además por me- 
Figura 3. Promedio de errores.

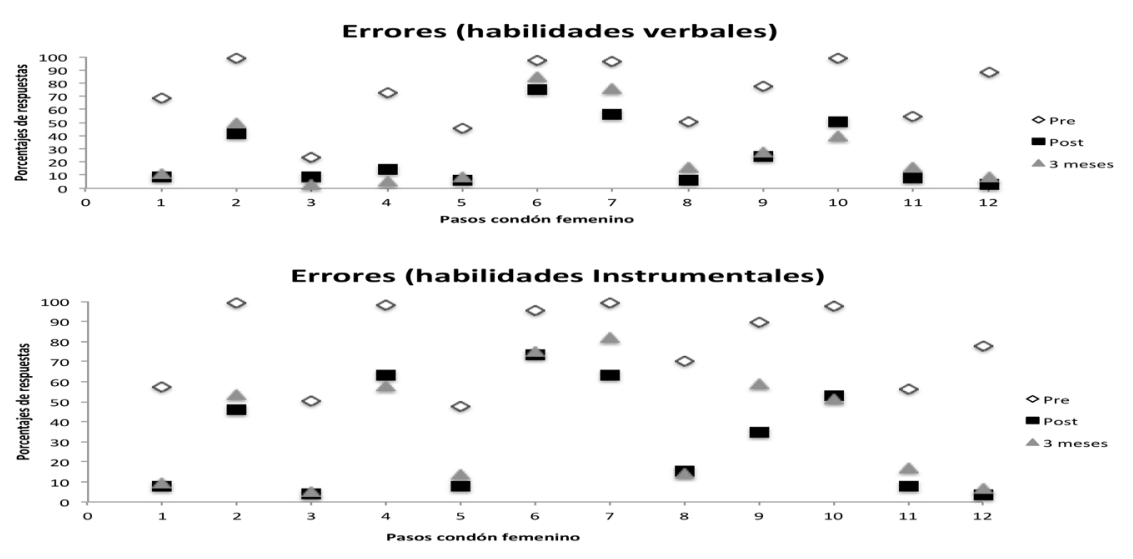

dio de vaginas de entrenamiento y condones. En efecto, diversos estudios sobre aprendizaje social han sugerido que el modelamiento es central en el aprendizaje de adolescentes y que parece estar estrechamente ligado en la forma en la cual despliegan conductas saludables y no saludables, incluyendo la conducta sexual (Whiteman, Zeiders, Killoren, Rodriguez y Updegraff, 2014). Este argumento puede ayudar a explicar por qué esta población se benefició de este tipo de entrenamiento, si bien se necesitan más investigaciones al respecto. De corroborarse los datos aquí obtenidos, el entrenamiento del condón femenino por medio del modelamiento implicaría una opción viable, rápida y económica para el desarrollo de habilidades del uso correcto del CF2 por la población adolescente.

Es de destacarse que los efectos obtenidos fueron muy similares en ambos tipos de entrenamiento, tanto para las habilidades verbales como instrumentales, y que estas se conservaron a través del tiempo, resultando estadísticamente significativas aun después de tres meses de seguimiento. Tal porción de los hallazgos es consistente con los de Hoffman et al. (2003), Choi et al. (2008) y Campbell et al. (2011) en población adulta. Sin embargo, los datos del presente estudio también mostraron que, pese a que los entrenamientos incrementaron el promedio de respuestas verbales e instrumentales, dicho promedio fue menor durante el seguimiento, en comparación con los resultados de la postevaluación. Lo anterior puede indicar la relevancia de instrumentar estrategias a guisa de recordatorio entre la postevaluación y el seguimiento para apoyar el mantenimiento de por- centajes más altos de respuestas verbales e instrumentales en el uso correcto del CF2.

En la presente investigación, los datos mostraron que los entrenamientos aumentaron el número de pasos correctos para utilizar el condón femenino. En estudios previos se ha sugerido que los entrenamientos conductuales en el uso del condón femenino probablemente fomentan en los participantes más habilidades y mejores actitudes hacia su uso (Campbell et al., 2011; Choi et al., 2008; Kalichman, et al., 1999; Van Devanter et al., 2002; Witte et al., 2006; Zimmers et al., 1999). También se ha sugerido una disminución de los actos sexuales no protegidos (Hoffman et al., 2003); dichos efectos han mostrado una tendencia a mantenerse incluso a los tres y seis meses una vez terminada la intervención. Sin embargo, muy poco o nada se ha reportado de la forma en que se usa el condón durante tales seguimientos. Con los datos obtenidos en el presente estudio parece claro que, aunque se incrementan las habilidades para usar correctamente el condón, estas aún distan de llegar al cien por ciento. Por lo anterior, es factible suponer que las personas que reciben los entrenamientos sí estén usando actualmente el condón femenino, aunque cometiendo tal vez algunos errores en su uso. Se considera que es indispensable que en futuras investigaciones no únicamente se indague si usan o no el condón, sino que evalúen cómo lo están utilizando y qué errores podrían estar cometiendo. Se encontró asimismo que los porcentajes de error disminuyeron en la postevaluación y en el seguimiento; no obstante, en algunos pasos ( 5 y 7, véase la Tabla 2) hubo mayor frecuencia de errores en dichas fases. Identificar los errores más comunes 
permitiría conocer los que ocurren con más frecuencia durante el acto coital, que, en el caso del condón masculino, por ejemplo, son las rupturas o los deslizamientos del dispositivo (Baćak y Stulhofer, 2012; Sanders et al., 2012). En el caso del condón femenino, los pasos apropiados para colocarlo y proteger adecuadamente los labios vaginales parecen ser particularmente desafiantes para los adolescentes, por lo que se recomienda que en trabajos futuros se dé especial énfasis a las estrategias que hagan posible aprender de manera más precisa dichos pasos. Se sugiere también que los participantes tengan la oportunidad de manipular previamente las vaginas de entrenamiento, pues algunas de ellas pueden mostrar cierta resistencia para colocarse de una manera sencilla y fluida. Es posible que esta sea una de las razones por las que en los pasos 6 y 7 hubo más errores al momento de llevarse a cabo las habilidades instrumentales, no así las verbales.

Llama la atención que en la preevaluación se hayan encontrado diferencias estadísticamente significativas debidas al sexo en las habilidades verbales para el uso correcto del CF2. Una posible explicación de lo anterior puede ser que, según los comentarios de los participantes, un par de meses antes del inicio de la presente investigación se impartió en la institución un taller de sexualidad femenina dirigido exclusivamente a mujeres, en el que, entre otros temas, se abordó la protección sexual a través de diversos métodos de protección, incluido el CF2, únicamente por medio de información general. Lo anterior podría ser la razón de que las mujeres del grupo de modelamiento tuvieran un mayor promedio de respuestas verbales en la preevaluación al referir el uso correcto del CF2, pero no de respuestas instrumentales. Tal diferencia desapareció una vez que los adolescentes fueron expuestos a alguna de las intervenciones.

A pesar del cuidado con el que se diseñó el estudio, es posible identificar algunas limitaciones que deben tenerse en cuenta en futuros trabajos. Si bien algunos estudios han recomendado repetir en al menos tres ocasiones la inserción del condón en modelos pélvicos antes de tomar en cuenta las evaluaciones (lo anterior con la finalidad de habituar al participante para que pueda posteriormente aplicarlo a la pareja o a modelos pélvicos [Choi et al., 2008; Witte et al., 2006]), las limitaciones de recursos y tiempo para brindar el entrenamiento en la escuela no permitieron instrumentar tal recomendación en la presente investigación.

Otra limitación es que el uso correcto del CF2 únicamente se entrenó en cuanto a las habilidades verbales e instrumentales requeridas, pero no se evaluó la actitud de los participantes hacia su uso o su posible uso en relaciones coitales reales. Investigaciones futuras podrían evaluar dichas variables, ya que se consideran importantes para ayudar de manera efectiva a prevenir ITS, incluidos el VPH y el VIH (Mantell et al., 2011). Debido a los objetivos originales planteados para el presente estudio, si bien se mostró que los entrenamientos aumentaron el número de pasos correctos para utilizar adecuadamente el CF2, no se hizo énfasis en su uso consistente. Sería importante que futuras investigaciones se enfocaran en evaluar algunas variables relacionadas con el uso consistente del condón (cfr. Moyo, Levandowski, MacPhail, Rees y Pettifor, 2008). Además, se recomienda promover el desarrollo de las habilidades de negociación, toda vez que diversos estudios han documentado que dicha variable puede ser conflictiva en la pareja e impedir el uso del condón masculino o el femenino (Chakrapani, Newman, Shunmugam y Dubrow, 2010; Schmid, 2012; Schmid, Leonard, Ritchie y Gwadz, 2015; Schuyler et al., 2016; Telles-Dias, Souto y Page-Shafer, 2006). Por la falta de los recursos humanos suficientes, un solo observador registró las respuestas de los estudiantes, por lo cual no fue posible obtener un índice de confiabilidad entre observadores; aunque este se entrenó exhaustivamente en el registro de los comportamientos, lo recomendable es que hubiera un cotejo o comparación para obtener ese índice (Campbell et al., 2011), por lo que se recomienda que en estudios futuros se supere tal limitación.

Otro punto que merece abordarse aquí es que después de haber finalizado el entrenamiento algunos participantes mencionaron que les había parecido adecuada la intervención; no reportaron que les produjera alguna molestia o inconveniente, y explícitamente comentaron que era una buena idea que se integrara al sexo masculino al entrenamiento del uso correcto del CF2, ya que habitualmente eran excluidos cuando se daba ese tipo de información. En efecto, no parece haber razón alguna que impida que los varones reciban el entre- 
namiento conductual en el uso correcto del CF2. Como lo muestran los datos, podrían verse igualmente beneficiados al utilizar estos programas, por lo que se recomienda que, independientemente del sexo del participante, futuras investigaciones entrenen y evalúen el uso del CF2 en virtud de sus probables ventajas, tal y como ocurre con el condón masculino (Orr et al., 1996; Shepherd et al., 2010).

Durante la fase de reclutamiento del estudio, más de una tercera parte de los adolescentes declinó participar en la investigación. Aunque una evaluación de los factores que influyeron en tal declinación o en el rechazo a entregar el formato de consentimiento informado no estaba dentro de los objetivos de este estudio, futuras investigaciones podrían identificar los factores de tipo sociodemográfico, cultural y las creencias de los adolescentes o de sus padres, responsables de ese hecho.

A pesar de las limitaciones descritas, es de subrayarse la importancia de los hallazgos del presente trabajo al fomentar investigación sistemática, conductual, sobre el uso correcto del CF2, en una población que tradicionalmente olvidada, misma que se puede beneficiar de los entrenamientos conductuales para desarrollar en ellos habilidades verbales e instrumentales que la protejan en su vida sexual.

\section{REFERENCIAS}

Baćak, V. y Stulhofer, A. (2012). Condom use errors and problems in a national sample of young Croatian adults. Archives of Sexual Behavior, 41(4), 995-1003. doi: 10.1007/s10508-011-9838-x.

Beksinska, M.E., Piaggio, G., Smit, J.A., Wu, J., Zhangy, P., Pienaar, J. y Joanis, C. (2013). Performance and safety of the second-generation female condom (FC2) versus the woman's, the VA worn-of-women, and the Cupid female condoms: A randomised controlled non-inferiority crossover trial. The Lancet. Global Health, 1(3), e146-e152. Disponible en línea: http://doi.org/10.1016/S2214-109X(13)70054-8.

Campbell, A.N.C., Tross, S., Hu, M.-C., Pavlicova, M., Kenney, J. y Nunes, E.V. (2011). Female condom skill and attitude: results from a NIDA Clinical Trials Network gender-specific HIV risk reduction study. AIDS Education and Prevention, 23(4), 329-340.

Cavazos-Rehg, P.A., Krauss, M.J., Spitznagel, E.L., Schootman, M., Bucholz, K.K., Peipert, J.F. y Bierut, L.J. (2009). Age of sexual debut among US adolescents. Contraception, 80(2), 158-162.

Chakrapani, V., Newman, P.A., Shunmugam, M. y Dubrow, R. (2010). Prevalence and contexts of inconsistent condom use among heterosexual men and women living with HIV in India: Implications for prevention. AIDS Patient Care and STDs, 24(1), 49-58.

Choi, K.-H., Hoff, C., Gregorich, S.E., Grinstead, O., Gomez, C. y Hussey, W. (2008). The efficacy of female condom skills training in HIV risk reduction among women: A randomized controlled trial. American Journal of Public Health, 98(10), 1841-1848.

Female Health Company (2012, agosto 26). All about the FC2 female condom. Chicago, Il: FHC. Disponible en línea: http:/femalehealth.com/wp-content/uploads/2016/05/All-About-the-FC2-female-condom.pdf.

Hoffman, S., Exner, T.M., Leu, C.-S., Ehrhardt, A.A. y Stein, Z. (2003). Female-condom use in a gender-specific family planning clinic trial. American Journal of Public Health, 93(11), 1897-1903.

Kalichman, S.C., Williams, E. y Nachimson, D. (1999). Brief behavioural skills building intervention for female controlled methods of STD-HIV prevention: Outcomes of a randomized clinical field trial. International Journal of STD \& AIDS, 10(3), 174-181.

Liu, G., Hariri, S., Bradley, H., Gottlieb, S.L., Leichliter, J.S. y Markowitz, L.E. (2015). Trends and patterns of sexual behaviors among adolescents and adults aged 14 to 59 years, United States. Sexually Transmitted Diseases, 42(1), 20-26.

Lopez, L.M., Stockton, L.L., Chen, M., Steiner, M.J. y Gallo, M.F. (2014). Behavioral interventions for improving dual-method contraceptive use. The Cochrane Database of Systematic Reviews, 3, CD010915. Disponible en línea: http://doi. org/10.1002/14651858.CD010915.pub2.

Mantell, J.E., Smit, J.A., Beksinska, M., Scorgie, F., Milford, C., Balch, E. y Stein, Z.A. (2011). Everywhere you go, everyone is saying condom, condom. But are they being used consistently? Reflections of South African male students about male and female condom use. Health Education Research, 26(5), 859-871.

Mantell, J.E., Smit, J.A., Exner, T.M., Mabude, Z., Hoffman, S., Beksinska, M. y Stein, Z.A. (2014). Promoting female condom use among female university students in KwaZulu-Natal, South Africa: Results of a randomized behavioral trial. AIDS and Behavior, 19(7), 1129-1140.

Moyo, W., Levandowski, B.A., MacPhail, C., Rees, H. y Pettifor, A. (2008). Consistent condom use in South African youth's most recent sexual relationships. AIDS and Behavior, 12(3), 431-440. 
Orr, D.P., Langefeld, C.D., Katz, B.P. y Caine, V.A. (1996). Behavioral intervention to increase condom use among high-risk female adolescents. The Journal of Pediatrics, 128(2), 288-295.

Sanders, S.A., Yarber, W.L., Kaufman, E.L., Crosby, R.A., Graham, C.A. y Milhausen, R.R. (2012). Condom use errors and problems: a global view. Sexual Health, 9(1), 81-95.

Schmid, A. (2012). Assertiveness during condom negotiation among high risk late adolescent/emerging adult couples: The role of relational uncertainty. Dissertation Abstracts International. Section B: The Sciences and Engineering, 73(6-B), 4001.

Schmid, A., Leonard, N.R., Ritchie, A.S. y Gwadz, M.V. (2015). Assertive communication in condom negotiation: Insights from late adolescent couples' subjective ratings of self and partner. Journal of Adolescent Health, 57(1), 94-99.

Schuyler, A.C., Masvawure, T.B., Smit, J.A., Beksinska, M., Mabude, Z., Ngoloyi, C. y Mantell, J.E. (2016). Building young women's knowledge and skills in female condom use: lessons learned from a South African intervention. Health Education Research, 31(2), 260-272.

Shepherd, J.P., Frampton, G.K. y Harris, P. (2011). Interventions for encouraging sexual behaviours intended to prevent cervical cancer. The Cochrane Database of Systematic Reviews (4), CD001035. Disponible en línea: http://doi.org/10.1002/14651858. CD001035.pub2.

Shepherd, J., Kavanagh, J., Picot, J., Cooper, K., Harden, A., Barnett-Page, E. y Price, A. (2010). The effectiveness and cost-effectiveness of behavioural interventions for the prevention of sexually transmitted infections in young people aged 13-19: a systematic review and economic evaluation. Health Technology Assessment, 14(7), 1-206.

Tapia A., V., Arillo S., E., Allen, B., Angeles L., A., Cruz V., A. y Lazcano P., E. (2004). Associations among condom use, sexual behavior, and knowledge about HIV/AIDS. A study of 13,293 public school students. Archives of Medical Research, 35(4), 334-343.

Telles-Dias, P.R., Souto, K. y Page-Shafer, K. (2006). Long-term female condom use among vulnerable populations in Brazil. AIDS and Behavior, 10(4 Suppl), S67-S75.

Van Devanter, N., Gonzales, V., Merzel, C., Parikh, N.S., Celantano, D. y Greenberg, J. (2002). Effect of an STD/HIV behavioral intervention on women's use of the female condom. American Journal of Public Health, 92(1), 109-115.

Vijayakumar, G., Mabude, Z., Smit, J., Beksinska, M. y Lurie, M. (2006). A review of female-condom effectiveness: patterns of use and impact on protected sex acts and STI incidence. International Journal of STD \& AIDS, 17(10), 652-659.

Whiteman, S.D., Zeiders, K.H., Killoren, S.E., Rodriguez, S.A. y Updegraff, K.A. (2014). Sibling influence on Mexican-origin adolescents' deviant and sexual risk behaviors: the role of sibling modeling. The Journal of Adolescent Health, 54(5), 587-592.

Witte, S.S., El-Bassel, N., Gilbert, L., Wu, E., Chang, M. y Hill, J. (2006). Promoting female condom use to heterosexual couples: findings from a randomized clinical trial. Perspectives on Sexual and Reproductive Health, 38(3), 148-154.

Zimmers, E., Privette, G., Lowe, R.H. y Chappa, F. (1999). Increasing use of the female condom through video instruction. Perceptual and Motor Skills, 88(3 Pt 2), 1071-1077. 